\title{
Case of paediatric relapsing polychondritis with severe airway involvement: the challenges of long- term airway and respiratory management
}

\author{
Trishul Kothari, ${ }^{1}$ Theodoros Valsamakis, ${ }^{2}$ Arani V Sridhar, ${ }^{1}$ Molla Imaduddin Ahmed
}

'Paediatrics, Leicester Childrens' Hospital, Leicester, UK ${ }^{2}$ ENT, Leicester Royal Infirmary, Leicester, UK

\section{Correspondence to} Dr Molla Imaduddin Ahmed: drahmed38@gmail.com

Accepted 1 August 2021
Check for updates

(c) BMJ Publishing Group Limited 2021. No commercial re-use. See rights and permissions. Published by BMJ.

To cite: Kothari T, Valsamakis T, Sridhar AV, et al. BMJ Case Rep 2021:14:e239774. doi:10.1136/bcr-2020239774

\section{SUMMARY}

We describe a case of a 15-year-old girl diagnosed with relapsing polychondritis (RP) with involvement of the tracheobronchial tree, resulting in an increased difficulty in breathing, hoarseness of voice and stridor. Her case required the input of multiple specialities including ear, nose and throat, rheumatology, respiratory team and intensive care. Airway assessment and imaging showed glottic and subglottic stenosis and left bronchomalacia. Despite the use of steroids, cyclophosphamide and rituximab, her symptoms progressed and she was started on overnight noninvasive ventilation.

She had further relapses of her airway RP — she was started on adalimumab and methotrexate and underwent monthly balloon dilatations which helped with her symptoms and facilitated a safe discharge home. However, she had further relapses and intensive care admissions and following further discussions, a tracheostomy was inserted. She is now stable on her tracheostomy and is off any respiratory support.

\section{BACKGROUND}

Relapsing polychondritis (RP) is a rare multisystem inflammatory disease mainly affecting cartilaginous structures of the ears, nose and tracheobronchial tree. The inner ear, eyes and cardiovascular system may be affected. ${ }^{1}$ Diagnosis is predominantly obtained clinically, with investigations and imaging, or occasionally cartilage biopsy providing supporting evidence. There are no current markers for the disease or its progression. ${ }^{2-4}$

Airway involvement may be diffuse or localised and may involve the upper extrathoracic airway, intrathoracic trachea or even bronchi. ${ }^{5} 6$ These children can present with increasing hoarseness of voice, stridor and increased respiratory distress which can be refractory to conventional treatment of steroids and nebulisers leading to a diagnostic dilemma.

Although RP with severe airway obstruction has been reported, there is limited literature on managing the long term respiratory challenges that occur with RP in children. We report a case of RP with severe airway involvement in a 15 -year-old child, and aim to highlight the challenges of airway and respiratory management in this child.

\section{CASE PRESENTATION}

A 15-year-old girl of African origin presented to the emergency department with a 6-week history of chest pain, 3 weeks of a red ear and red eyes, 4 weeks of night sweats and $5 \mathrm{~kg}$ weight loss over 8 weeks. She was diagnosed with right ear perichondritis and managed with intravenous antibiotics, however, due to continued fever spikes and arthritis of both knee joints, bilateral uveitis and mild sensori-neuronal deafness, she was discussed with the tertiary paediatric rheumatology team who suspected RP and advised to start on a high dose of steroids.

Three months later, she was readmitted with an episode of increasing hoarseness of voice and stridor suggestive of a progression of RP involving the tracheobronchial tree. She was started on intravenous methylprednisolone and treated with budesonide and epinephrine nebulisers. Flexible nasal endoscopy showed erythematous vocal cords and epiglottis. She was started on steroids and disease modifying antirheumatic drugs (DMARDs) - her symptoms improved and she was discharged home.

One month later, the patient presented to the hospital with respiratory distress and worsening biphasic stridor, especially during sleep. CT scan (CT) of neck and thorax (figure 1) showed changes consistent with RP with focal obliteration of the airway at the level of supraglottic, glottic and subglottic area and moderate narrowing of the left main bronchus. CT bronchogram (figure 2) showing narrowing of the left main bronchus at zero positive end expiratory pressure (PEEP); this was noted to improve on a PEEP of $12 \mathrm{~cm} \mathrm{H} 2 \mathrm{O}$-this was on non-invasive ventilation (NIV) using NIPPY junior ventilator.

She had subsequent worsening biphasic stridor necessitating paediatric intensive care unit (PICU) admission with a relapse of her airway RP. Repeat CT neck scan (figure 3 ) was similar to previous changes showing severe narrowing at the level around the glottis. Despite optimal management with intravenous steroids, heliox and increase in respiratory support, her respiratory status worsened, and she was intubated-the paediatric anaesthetic team were only able to introduce a size 5.0 cuffed endotracheal tube (ETT) in theatre. She remained ventilated for 24 hours and was then able to mobilise with an ETT and Swedish nose in situ. (A 'Swedish' nose is otherwise known as a heat moisture exchanger, is a filter that can be attached to the tracheostomy tube and comes in several shapes and sizes. This is also known by several other terms including; thermal humidifying filters, artificial nose, filter and thermovent). 

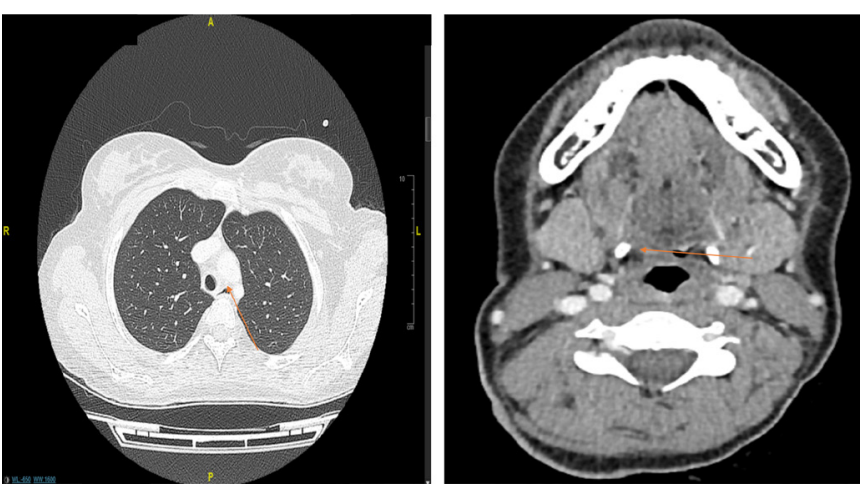

Figure 1 CT neck and thorax — changes consistent with known relapsing with focal obliteration of the airway at the level of the glottis/ subglottis and moderate narrowing of the left main bronchus.

The patient attended the operating theatre for a microlaryngobronchoscopy (MLB) where she had an awake fibreoptic intubation. Once her airway was secured, her lower airway was assessed via the ETT with a fibreoptic bronchoscope and the use of Storz recording system attached for documentation. Gross anatomy of the hypopharynx and laryngeal inlet was assessed initially while the patient remained intubated. Subsequently, following detailed briefing of back up plans including reintubation sequence and tracheostomy, the patient was briefly extubated and supported via a nasopharyngeal airway. This allowed a narrow but sufficient time to assess the remaining of the glottis and subglottic level prior to reintubation required. Appropriate size equipment, $2.7 \mathrm{~mm}, 0^{\circ}$ and $30^{\circ}$ Hopkins rod rigid scopes, were used throughout the procedure. Her MLB (figure 4) showed a floppy epiglottis, bulky appearance of arytenoids, swollen vocal cords and left severe bronchomalacia. The right main bronchus and trachea were normal. Appearances were consistent with chronic changes secondary to RP.

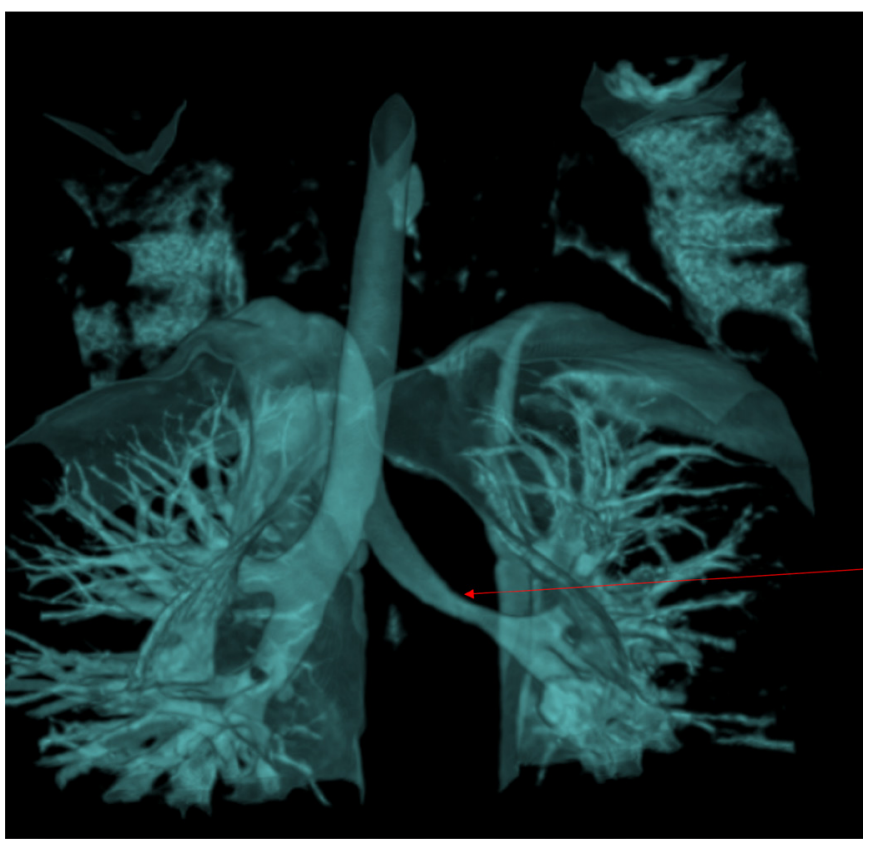

Figure 2 CT bronchogram - the left main bronchus demonstrates narrowing with PEEP of zero. PEEP of 12 and 15 demonstrate wide patency of the left main bronchus. PEEP, peak end expiratory pressure.

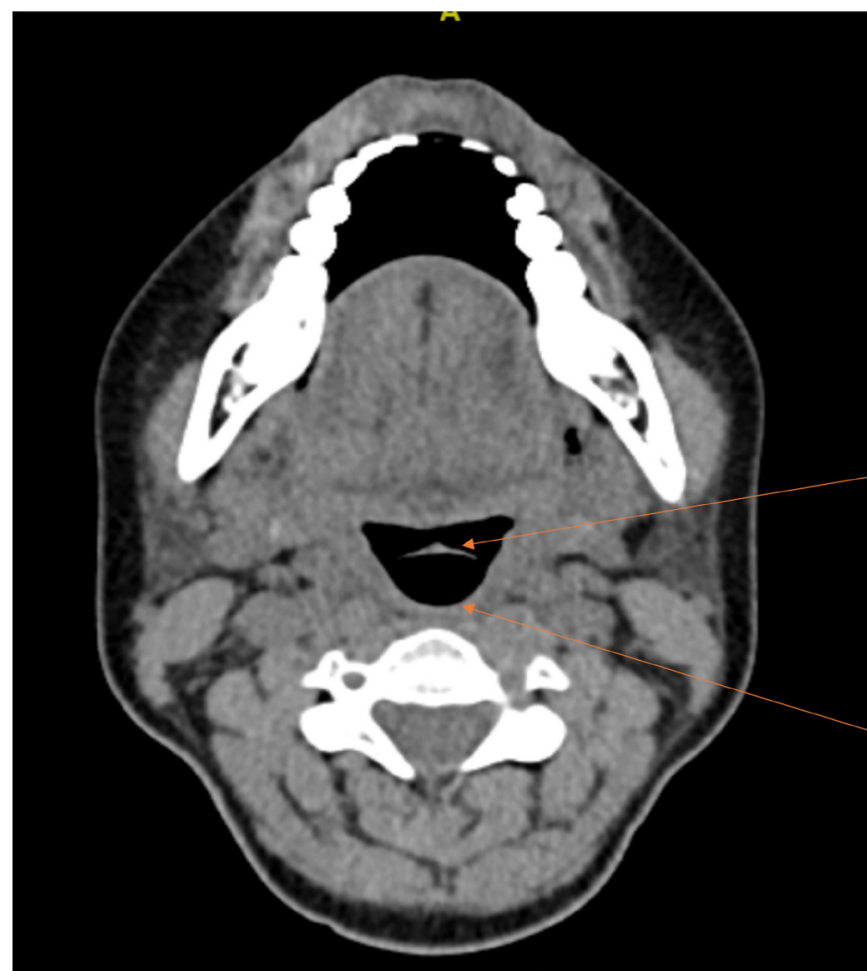

Figure 3 CT neck showing similar appearances to the prior study with severe airway narrowing at the level of the glottis.

The consensus from the respiratory, ear, nose and throat (ENT) and PICU teams was that although tracheostomy and NIV could be a safe long-term option to maintain her airway, it was in child's best interest to explore any other possible management options. She was continued on overnight bilevel positive airways pressure (BiPAP) and was transferred to a quaternary specialist paediatric airway centre for further opinion. The multi-disciplinary team (MDT) at the quaternary centre felt that it was not in her best interest to have a tracheostomy,tracheal stenting or reconstructive surgery at that time. A further cardiorespiratory sleep study was performed showing ongoing obstruction and hypoventilation and she was continued on BiPAP.

She had a few further relapses of her airway RP, which were managed with pulses of intravenous steroids and escalation of her respiratory support. She thereafter had an episode of respiratory exacerbation which was refractory to conservative management and led to 'in theatre' intubation with a size five uncuffed ETT. At that time, MLB confirmed severe subglottic oedema (figure 5) as well as severe bronchomalacia, which was already known from the previous assessment. In view of this, options were discussed with the child and her mum-tracheostomy was discussed, however, both mum and patient were not keen on this as an elective choice. They agreed though, that a tracheostomy may be needed in an emergency situation to secure her airway. She was managed with monthly balloon dilatations with continuation of overnight NIV.

Six weeks later, she was reviewed in the respiratory clinicher exercise tolerance tests showed significantly reduced tolerance. Her spirometry showed forced expiratory volume in $1 \mathrm{~s}$ of $1.34 \mathrm{~L}$ (43\% predicted) and forced vital capacity $1.97 \mathrm{~L} \mathrm{(56 \%}$ predicted). She was admitted for repeat balloon dilation of her trachea, however, the procedure was complicated due to severe worsening subglottic oedema resulting in reintubation. An MDT meeting was organised that involved the paediatric intensivists, 


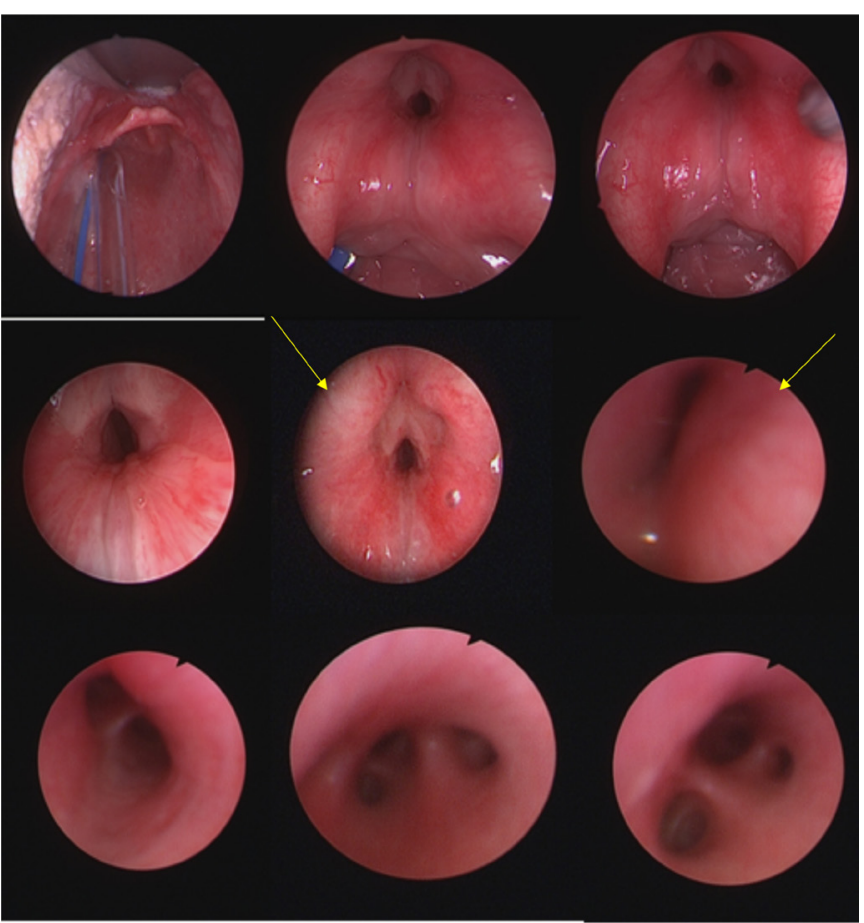

Figure 4 MLB shows floppy epiglottis, bulky appearance of arytenoids filling piriform fossa, swollen cords less discrete at anterior commissure. There is left severe bronchomalacia while right main bronchus and trachea are normal. MLB, microlaryngobronchoscopy.

ENT, respiratory and rheumatology teams, the consensus was to offer her a tracheostomy. However, child and her mother refused that option and opted to continue with conservative approach. She was discharged with a plan for monthly balloon dilatations and overnight BiPAP.

Three months later, she represented with respiratory deterioration needing intubation. Following discussion with mother and

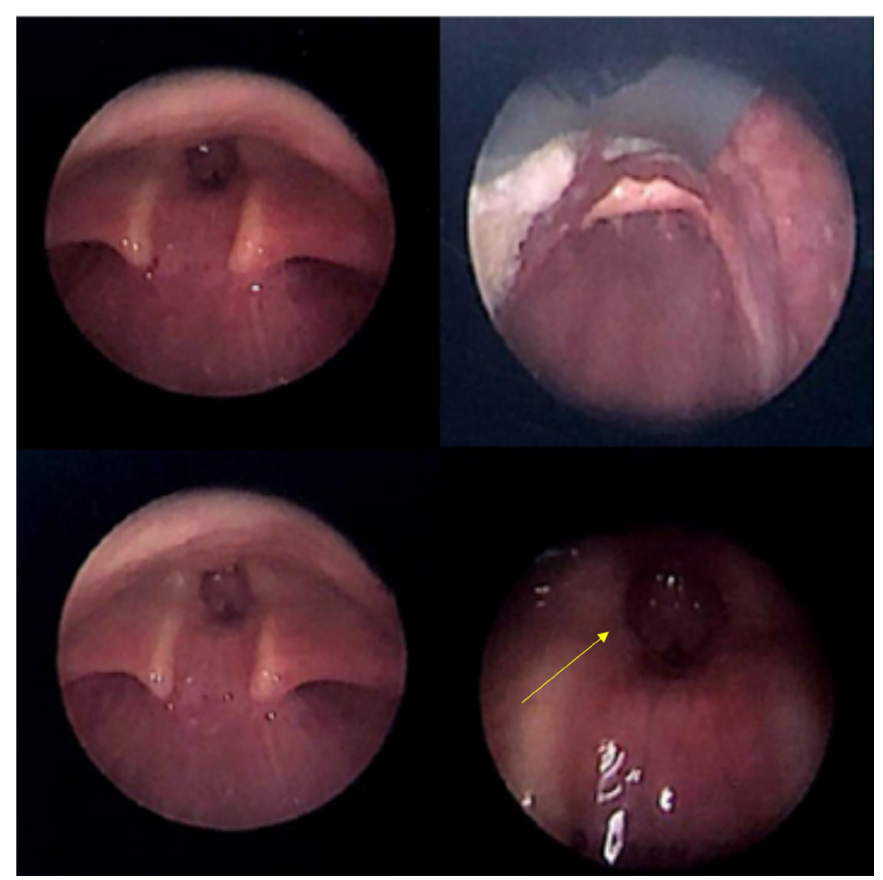

Figure 5 MLB showing severe subglottic oedema. MLB, microlaryngobronchoscopy. child, a tracheostomy was inserted. Her intensive care stay was complicated with episodes of poor oxygenation and subsequent pneumothoraces, which led to reintubation and tracheostomy removal. Revision tracheostomy took place at the quaternary children's hospital. MLB during that session showed grade 3 subglottic stenosis, localised granulation tissue and she underwent a further balloon dilatation.

She was transferred back to the local centre following her tracheostomy insertion. Her overnight respiratory support has been gradually weaned off. She is now stable with her tracheostomy with no clinical concerns and has been off her overnight respiratory support for last 9 months.

\section{INVESTIGATIONS}

Her blood tests during her initial presentation showed a raised $\mathrm{C}$ reactive protein of $100 \mathrm{mg} / \mathrm{L}$, a raised plasma viscosity of 2.64 millipascal seconds (upper limit 1.7) and a normal autoimmunity screen. CT of neck and thorax (figure 1) showed changes consistent with RP with focal obliteration of the airway at the level of of supraglottic, glottic and subglottic area. CT bronchogram (figure 2) showing narrowing of the left main bronchus at zero PEEP, this was noted to improve on a PEEP of $12 \mathrm{~cm}$. A cardiorespiratory sleep study was performed showing ongoing obstruction and hypoventilation. MLB prior to tracheostomy during showed grade 3 subglottic stenosis, localised granulation tissue as well as severe left bronchomalacia.

\section{DIFFERENTIAL DIAGNOSIS}

RP can be mimicked by granulomatosis with polyangitis, T-cell lymphoma, sarcoidosis. ${ }^{2}$ Thirty per cent of the time it can be associated with Systemic lupus erythematosus (SLE), Sjogrens, vasculitis, antiphospholipid syndrome, rheumatoid arthritis, spondyloarthritis, inflammatory bowel disease, thyroiditis and others. $^{7}$

\section{TREATMENT}

As well as her airway management outlined above, we outline here her medical therapy management for RP. On her initial presentation with RP, she was treated with high dose of steroids daily ( $1 \mathrm{mg} / \mathrm{kg} / \mathrm{dose})$ and was discharged home with a weaning steroid plan. Her acute episodes of relapses of airway RP have been treated with high-dose intravenous methylprednisolone, epinephrine nebulisers and escalation of her respiratory support. To prevent flare-ups of her disease, she was started on a treatment regime, which comprised high-dose prednisolone, cyclophosphamide and rituximab with good improvement in her symptoms. Following her review at the quaternary centre, she was continued on prednisolone, cyclophosphamide and transferred back for continuing care with a plan of 1 month weaning dose of prednisolone and completion of her cyclophosphamide course. Due to further multiple relapses, her RP management was escalated to adalimumab and subcutaneous methotrexate, which was commenced after completion of seven cycles of cyclophosphamide. Due to prolonged steroid therapy, she has evidence of multiple adverse effects including weight gain, osteopenia and low mood.

\section{OUTCOME AND FOLLOW-UP}

She has a tracheostomy size seven uncuffed Bivona and has been stable off overnight respiratory support for last 9 months with no clinical concerns. She continues on a slow weaning regimen of prednisolone, weekly subcutaneous methotrexate and 2 weekly subcutaneous adalimumab to prevent flare-ups of the disease. 
Discharge planning is ongoing with tracheostomy training for her carers.

\section{DISCUSSION}

$\mathrm{RP}$ is a very rare disease with an annual incidence estimated at 3.5 per million persons, with a usual onset at over 40 years of age. Less than $5 \%$ cases are seen in paediatrics. ${ }^{158}$

Early diagnosis and initiation of treatment can alter the natural course and prevent worsening. It can take 3 years between manifestation of first symptom and diagnosis. ${ }^{7}$ Diagnosis is very difficult and can be missed, as it remains largely based on clinical findings.

The classification criteria include involvement of at least two out of three of the auricular, nasal or laryngotracheal cartilages ${ }^{7}$; diagnosis can also be made if proven inflammation in one cartilage as well as other signs including ocular inflammation, vestibular dysfunction, seronegative inflammatory arthritis or hearing loss. ${ }^{9}$ The cause is unknown but regarded as immune mediated and there is a strong association with HLA DR4 (Human Leucocyte antigen DR4 serotype) in adults. ${ }^{10}$

Airway management is a complex issue in people with RP, $25 \%$ of cases will develop laryngotracheal stricture. ${ }^{11}$ There is a risk of tracheal perforation with MLB. ${ }^{12}$ Tracheal narrowing may require tracheal dilatation, stenting, tracheostomy and reconstructive surgery but these procedures can aggravate RP and can cause distal inflammation. ${ }^{12}$ Various case reports in adults have demonstrated the potential successes of tracheostomy and bronchoscopic stenting, although with lower respiratory tract infections being reportedly common, these are prone to being blocked by thick secretions. ${ }^{13-16} \mathrm{~A}$ risk of pneumonia remains problematic with these patients due to reduced airway secretion clearance. ${ }^{15}$ Major airway collapse may occur suddenly and cause rapid death. Survival rates of $74 \%$ at 5 years and $55 \%$ at 10 years in adults, no similar data in children. ${ }^{1718}$

From initial presentation to her current status, the various teams in our centre have been able to perform the appropriate investigations such as CT bronchogram (figure 1) and MLB (figure 4) successfully while mitigating the risks of possible harm to her airways.

The rate of progression of disease within 12 months in our patient has not been reported as widely in other cases. Management in children is generally more aggressive as RP is usually less benign in this age group. Intubation is generally avoided in these high-risk patients and is only undertaken is there is worsening respiratory failure refractory to non-invasive support and medical treatments such as epinephrine nebulisers and intravenous steroids. The optimal place to intubate these patients is the operating theatre with senior anaesthetists and ENT surgeons as in this case, the airway is likely to be narrowed due to inflammation. There may be risk of needing urgent tracheostomy and this equipment needs to be readily available.

Treatment ranges from use of non-steroidal antiinflammatories to DMARDs. A maintenance dose of corticosteroids is required to prevent relapses and increased dose of steroids are required intravenously during flare-ups. Emerging treatment options include the use of immunomodulatory agents such as cyclophosphamide, rituximab which were the chosen treatment of RP in our patient by the regional tertiary paediatric rheumatology team. ${ }^{15}$ Other agents include Ciclosporin, methotrexate and monoclonal antibodies. A recent systematic review of TNF - alpha (Tumour Necrosis actor - alpha) inhibitors has demonstrated improvement in health and respiratory symptoms in $67.8 \%$ of patients and $61 \%$ of patients, respectively. ${ }^{13}$
Complete remission of RP has been reported post autologous stem cell transplant in one case study to date. ${ }^{19}$

If medical therapy continues to fail in the progression of the RP, surgical intervention usually involves tracheostomy. Further surgical options like airway stenting can be considered and there are some case series reported in adults with RP. For example, a case series with adult patients in South Korea has shown effective use of endobronchial stents and tracheostomies in 2 out of 12 patients with RP and severe airway involvement, resulting in increased survival and airway improvement. ${ }^{14}$ Another case report from Japan involved the use of $\mathrm{T}$ tube stenting and the patient has reportedly disease control and symptom improvement. $^{15}$

Various case reports have demonstrated varying success with surgical procedures such as tracheostomy and stenting in management of airway RP but further evidence regarding outcome with balloon dilatation is very limited. ${ }^{13-15}$

This case highlights the importance of MDT management and requesting for specialist expertise in management of rare conditions. This also shows the importance of balancing the requests and needs of the child and family and the burden of care with the risks and benefits of a long-term management like tracheostomy.

\section{Learning points}

Relapsing polychondritis (RP) is rare with estimated incidence of 3.5 per million, less than $5 \%$ cases are seen in paediatrics. The rarity of the condition and the limitations to its diagnosis require high index of awareness.

- The lack of strong evidence on its management in fast progressing cases, like ours, highlights the need for a thorough multidisciplinary approach and involvement of specialist centres to provide the necessary expertise.

- Without aggressive and prompt treatment, life-threatening complications can develop rapidly, and outcomes can be poor.

- Managing RP in children with progressive significant airway involvement with both NIV and repeated balloon dilatations can help to defer surgical intervention and provide a novel approach for milder cases; however, tracheostomy may be required, and this should be discussed with parents and the child from the outset.

Acknowledgements We would like to acknowledge the following contributors to the management of this patient: 1. Miss Marianne Elloy-ENT consultant at University Hospitals of Leicester. 2. Paediatric Intensive Care team at University Hospitals of Leicester. 3. ENT team at Great Ormond Street Hospital and 4. Paediatric Rheumatology team at Birmingham Children's hospital.

Contributors TK : selection of case, drafting the mansucript, revised the manuscript and final approval. TV: selection of case, revised the manuscript and final approval. AVS : selection of case, revised the manuscript and final approval. MIA: selection of case, revised the manuscript and final approval.

Funding The authors have not declared a specific grant for this research from any funding agency in the public, commercial or not-for-profit sectors.

Competing interests None declared.

Patient consent for publication Obtained.

Provenance and peer review Not commissioned; externally peer reviewed.

ORCID iD

Molla Imaduddin Ahmed http://orcid.org/0000-0001-7777-5945

\section{REFERENCES}

1 Eng J, Sabanathan S. Airway complications in relapsing polychondritis. Ann Thorac Surg 1991;51:686-92. 
2 Horváth A, Páll N, Molnár K, et al. A nationwide study of the epidemiology of relapsing polychondritis. Clin Epidemiol 2016;8:211-30.

3 Knipp S, Bier H, Horneff $\mathrm{G}$, et al. Relapsing polychondritis in childhood--case report and short review. Rheumatol Int 2000;19:231-4.

4 Emmungil H, Aydin SZ. Relapsing polychondritis. Eur J Rheumatol 2015;2:155-9.

5 Ernst A, Rafeq S, Boiselle P, et al. Relapsing polychondritis and airway involvement. Chest 2009;135:1024-30.

6 Tillie-Leblond I, Wallaert B, Leblond D, et al. Respiratory involvement in relapsing polychondritis. Clinical, functional, endoscopic, and radiographic evaluations. Medicine 1998;77:168-76.

7 Rednic S, Damian L, Talarico R, et al. Relapsing polychondritis: state of the art on clinical practice guidelines. RMD Open 2018;4:e000788.

8 Gorard C, Kadri S. Critical airway involvement in relapsing polychondritis. BMJ Case Rep 2014;2014. doi:10.1136/bcr-2014-205036. [Epub ahead of print: 11 Sep 2014].

9 Mpofu S, Estrach C, Curtis J, et al. Treatment of respiratory complications in recalcitrant relapsing polychondritis with infliximab. Rheumatology 2003;42:1117-8.

10 Rosen O, Thiel A, Massenkeil G, et al. Autologous stem-cell transplantation in refractory autoimmune diseases after in vivo immunoablation and ex vivo depletion of mononuclear cells. Arthritis Res 2000;2:327-36.

11 Kent PD, Michet CJ, Luthra HS. Relapsing polychondritis. Curr Opin Rheumatol 2004:16:56-61.
12 Hazra N, Dregan A, Charlton J, et al. Incidence and mortality of relapsing polychondritis in the UK: a population-based cohort study. Rheumatology 2015;54:kev240-187.

13 Biya J, Dury S, Perotin J-M, et al. Assessment of TNF- $\alpha$ inhibitors in airway involvement of relapsing polychondritis: a systematic review. Medicine 2019;98:e17768.

14 Hong G, Kim H. Clinical characteristics and treatment outcomes of patients with relapsing polychondritis with airway involvement. Clin Rheumatol 2013:32:1329-35.

15 Nakayama T, Horinouchi H, Asakura K, et al. Tracheal stenosis due to relapsing polychondritis managed for 16 years with a silicon T-tube covering the entire trachea. Ann Thorac Surg 2011;92:1126-8.

16 Rafeq S, Trentham D, Ernst A. Pulmonary manifestations of relapsing polychondritis. Clin Chest Med 2010;31:513-8.

17 Tsunezuka Y, Sato H, Shimizu H. Tracheobronchial involvement in relapsing polychondritis. Respiration 2000;67:320-2.

18 Kawano T, Matsuse H, Kinoshita N, et al. Bronchoscopic observation of unusual deformities of bronchial cartilage and subsequent airway narrowing in respiratory relapsing polychondritis. Am J Case Rep 2012;13:114-7.

19 Suzuki S, Ikegami A, Hirota Y, et al. Fever and cough without pulmonary abnormalities on CT: relapsing polychondritis restricted to the airways. Lancet 2015;385:88-6736.

Copyright 2021 BMJ Publishing Group. All rights reserved. For permission to reuse any of this content visit https://www.bmj.com/company/products-services/rights-and-licensing/permissions/ BMJ Case Report Fellows may re-use this article for personal use and teaching without any further permission.

Become a Fellow of BMJ Case Reports today and you can:

- Submit as many cases as you like

- Enjoy fast sympathetic peer review and rapid publication of accepted articles

- Access all the published articles

Re-use any of the published material for personal use and teaching without further permission

Customer Service

If you have any further queries about your subscription, please contact our customer services team on +44 (0) 2071111105 or via email at support@bmj.com. Visit casereports.bmj.com for more articles like this and to become a Fellow 